SAGA-HE-198-03

October 1, 2003

\title{
Neutrino Scattering Physics at Superbeams and Neutrino Factories
}

\author{
S. Kumano * \\ Department of Physics \\ Saga University \\ Saga 840-8502, Japan
}

Plenary talk at the 5th International Workshop on

Neutrino Factories \& Superbeams

Columbia University, New York, USA

June 5-11, 2003 (talk on June 5)

*Email:kumanos@cc.saga-u.ac.jp. URL: http://hs.phys.saga-u.ac.jp. 


\title{
Neutrino Scattering Physics at Superbeams and Neutrino Factories
}

\author{
S. Kumano \\ Department of Physics, Saga University, Saga, 840-8502, Japan
}

\begin{abstract}
Neutrino scattering physics is discussed for investigating internal structure of the nucleon and nuclei at future neutrino facilities. We explain structure functions in neutrino scattering. In particular, there are new polarized functions $g_{3}, g_{4}$, and $g_{5}$, and they should provide us important information for determining internal nucleon spin structure. Next, nuclear structure functions are discussed. From $F_{3}$ structure function measurements, valence-quark shadowing should be clarified. Nuclear effects on the NuTeV $\sin ^{2} \theta_{W}$ anomaly are explained. We also comment on low-energy neutrino scattering, which is relevant to current long-baseline neutrino oscillation experiments.
\end{abstract}

\section{INTRODUCTION}

Nucleon structure has been investigated experimentally by various scattering experiments. Now, the perturbative QCD is well understood. The nonperturbative part is studied by theoretical models and lattice calculations, and they are tested experimentally. Because of these efforts, many aspects of the nucleon substructure are understood. However, there are still missing points. For example, spin is a fundamental quantity, and yet nucleon spin is poorly understood. We still do not know how the spin is constituted in terms of quarks and gluons. Future neutrino facilities should be able to provide important information on the internal hadron structure including the spin.

High-energy neutrino reactions have been already used for investigating the nucleon structure and determining fundamental constants such as the running coupling constant $\alpha_{S}$ and weak-mixing angle $\sin ^{2} \theta_{W}$. From accurate neutrino deep inelastic scattering (DIS) data, the structure functions, $F_{1}, F_{2}$, and $F_{3}$, have been extracted. Future neutrino facilities, superbeams [1] and neutrino factories [2], will provide new insight into the hadron substructure. Specialized talks are presented in the working group 2 (WG2) of this workshop, so that the details should be found in its summary [3] and presentations $[4,5,6,7]$. Neutrino beams are strong enough to allow proton and polarized targets at the considered neutrino factories. Therefore, the nucleon structure functions and the fundamental constants are obtained without worrying about nuclear corrections. In addition, it is important that polarized structure functions, especially new functions $g_{3}$, $g_{4}$, and $g_{5}$, could be measured. Using these polarized structure functions, we expect that the internal nucleon spin structure will be precisely understood.

The future neutrino facilities are supposed to contribute also to nuclear physics. In the present neutrino DIS, accurate measurements have been done mainly for the nuclear target, iron, so that neutrino-nucleus scattering data already exist. However, there is no accurate deuteron or proton data for investigating nuclear corrections in neutrino 
reactions by taking the ratio $\sigma_{v A} / \sigma_{v D}$. Because the proton and deuteron cross sections should be accurately measured at the future facilities, we could shed light on the nuclear corrections. In particular, measurements of the function $F_{3}$ will clarify the valence shadowing phenomenon. On the other hand, we could investigate nuclear effects such as Pauli exclusion and nucleon-nucleon correlation in the low-energy scattering.

This paper consists of the following. The unpolarized and polarized neutrino-nucleon scattering processes are explained, and then nuclear structure functions are discussed. We also comment on low-energy neutrino scattering. Finally, the discussions are summarized.

\section{UNPOLARIZED NEUTRINO-NUCLEON SCATTERING}

The cross section for unpolarized neutrino-nucleon DIS is calculated by assuming a oneboson exchange process, and then the charged-current (CC) cross section is expressed in terms of three structure functions, $F_{1}, F_{2}$, and $F_{3}$ :

$$
\frac{d^{2} \sigma_{C C}}{d x d y}=\frac{G_{F}^{2} s}{2 \pi\left(1+Q^{2} / M_{W}^{2}\right)^{2}}\left[x y^{2} F_{1}+(1-y) F_{2} \pm y(1-y / 2) x F_{3}\right] .
$$

Here, + and - of \pm indicate neutrino and antineutrino reactions, respectively, $G_{F}$ is the Fermi coupling constant, $s$ is the center-of-mass squared energy, $Q^{2}$ is defined by the momentum transfer $q: Q^{2}=-q^{2}$, and $M_{W}$ is the $W$ boson mass. The kinematical variables $x$ and $y$ are defined by $x=Q^{2} /\left(2 M q^{0}\right)$ and $y=q^{0} / E$ with the nucleon mass $M$ and the initial neutrino energy $E$. There are sum rules for these structure functions:

$$
\begin{aligned}
S_{A} & =\int_{0}^{1} \frac{d x}{x}\left[F_{2}^{\bar{v} p}\left(x, Q^{2}\right)-F_{2}^{v p}\left(x, Q^{2}\right)\right]=2, \\
S_{B j} & =\int_{0}^{1} d x\left[F_{1}^{v n}\left(x, Q^{2}\right)-F_{1}^{v p}\left(x, Q^{2}\right)\right]=1-\frac{2}{3} \frac{\alpha_{S}\left(Q^{2}\right)}{\pi}+\cdots+O\left(1 / Q^{2}\right), \\
S_{G R S} & =\frac{1}{2} \int_{0}^{1} d x\left[F_{3}^{\bar{v} p}\left(x, Q^{2}\right)+F_{3}^{v p}\left(x, Q^{2}\right)\right]=3\left[1-\frac{\alpha_{S}\left(Q^{2}\right)}{\pi}+\cdots\right]+O\left(1 / Q^{2}\right) .
\end{aligned}
$$

These are called Adler, unpolarized Bjorken, and Gross-Llewellyn Smith sum rules. There are perturbative QCD corrections to the last two sum rules, and they have been investigated up to the $\alpha_{s}^{4}$ level [8]. Therefore, sum-rule measurements will provide valuable information for an accurate determination of $\alpha_{s}$. Possible ambiguities come from the higher-twist corrections $O\left(1 / Q^{2}\right)$. Therefore, it is important to understand twist-four corrections theoretically, and such studies should be tested experimentally in the small- $Q^{2}$ region at the future neutrino facilities. The details of these points are summarized in the previous workshop [8].

The structure functions are expressed in terms of parton distribution functions (PDFs). The CC cross section is calculated in the parton model by using the current

$$
J_{C C}^{\mu}=\bar{u} \gamma^{\mu}\left(1-\gamma_{5}\right)\left[d \cos \theta_{c}+s \sin \theta_{c}\right]+\bar{c} \gamma^{\mu}\left(1-\gamma_{5}\right)\left[s \cos \theta_{c}-d \sin \theta_{c}\right]
$$


where $\theta_{c}$ is the Cabbibo angle. Comparing the obtained cross section with Eq. (1), we have the leading-order (LO) expressions for the structure functions in terms of the PDFs:

$$
\begin{gathered}
2 x\left(F_{1}^{v p}\right)_{C C}=\left(F_{2}^{v p}\right)_{C C}=2 x(\bar{u}+d+s+\bar{c}), \\
2 x\left(F_{1}^{\bar{v} p}\right)_{C C}=\left(F_{2}^{\bar{v} p}\right)_{C C}=2 x(u+\bar{d}+\bar{s}+c), \\
x\left(F_{3}^{v p}\right)_{C C}=2 x(-\bar{u}+d+s-\bar{c}), \quad x\left(F_{3}^{\bar{v} p}\right)_{C C}=2 x(u-\bar{d}-\bar{s}+c) .
\end{gathered}
$$

Neutron structure functions are obtained by using the isospin symmetry for the PDFs. Partonmodel expressions for neutral current (NC) structure functions are not shown here, but they are found, for example, in Refs. [9, 10].

Using the neutrino DIS data together with other lepton and hadron scattering data, we obtain the PDFs in the nucleon. The present situation is illustrated in Fig. 1 [11], where the MRST02 distributions are shown at $Q^{2}=10 \mathrm{GeV}^{2}$ as an example. Because these distributions are rather well

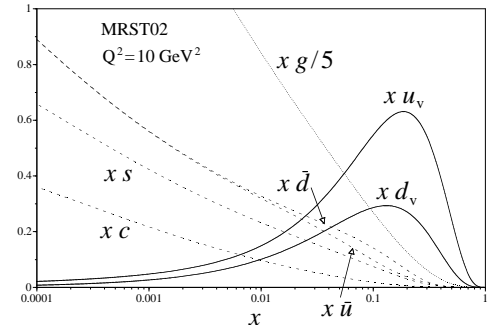

FIGURE 1. Parton distribution functions at $Q^{2}=10 \mathrm{GeV}^{2}$. determined, we had better focus on other aspects such as polarized and nuclear PDFs at future neutrino facilities.

\section{POLARIZED NEUTRINO-NUCLEON SCATTERING}

Polarized structure functions have been investigated by electron and muon DIS. Current polarized PDFs are determined by analyzing these data. Inclusive data are listed by the spin asymmetry $A_{1}$, which is expressed $A_{1} \cong$ $2 x(1+R) g_{1} / F_{2}$, where $R$ is the longitudinaltransverse structure function ratio and $g_{1}$ is a polarized structure function. The $g_{1}$ is given by the polarized PDFs which are expressed by a number of parameters. These parameters are determined by a $\chi^{2}$ analysis with the spin asymmetry data.

Recent analysis results are illustrated in Fig. 2 [12], where the polarized PDFs and their errors by Blümlein and Böttcher are shown as an example. The polarized valence-
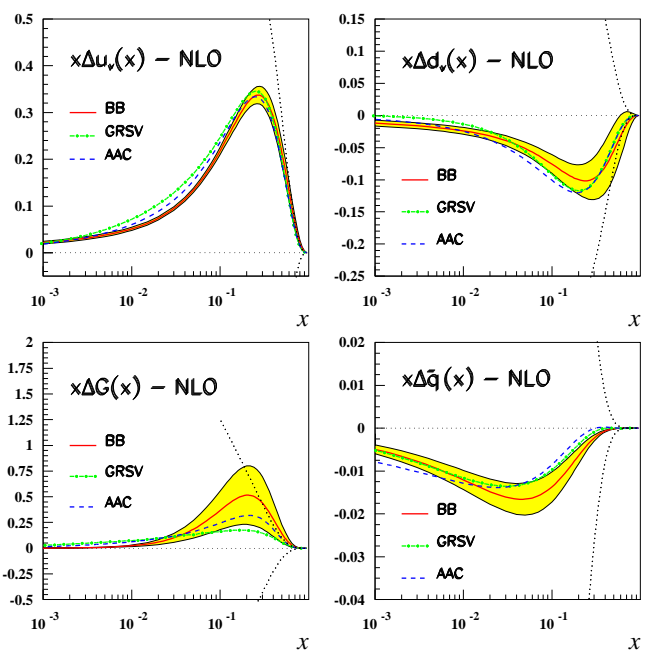

FIGURE 2. Recent polarized PDFs [12]. quark, antiquark, and gluon distributions are shown. Three different parametrization results are compared, and they agree each other except for the gluon distribution. The error bands for the valence-quark distributions are small; however, the error is large especially for the gluon distribution. It indicates that the polarized gluon distribution cannot be fixed at this stage. 
The results may seem to indicate that the polarized PDFs are rather well determined except for the gluon. However, there are important points to be investigated. The overall magnitudes of the polarized valence-quark distributions are fixed by low-energy seimileptonic decay data with a flavor symmetric assumption for the antiquark distributions $(\Delta \bar{u}=\Delta \bar{d}=\Delta \bar{s})$. Furthermore, the quark spin content $\Delta \Sigma$ cannot be determined from the current electron and muon DIS experiments although the analyses indicate a small fraction $\Delta \Sigma=10-30 \%$. These issues could be clarified by future neutrino DIS studies as explained in the following.

In addition to $g_{1}$ and $g_{2}$, there exist extra functions $g_{3}, g_{4}$, and $g_{5}$ in neutrino reactions. There are various definitions for $g_{3}, g_{4}$, and $g_{5}$ depending on researchers, so that one should be careful in reading related papers. In the following, we use the convention in Refs. $[2,13,14]$. The asymmetry $\Delta \sigma$ is the difference between polarized cross sections: $\Delta \sigma=\sigma_{\lambda_{p}=-1}-\sigma_{\lambda_{p}=+1}$, where $\lambda_{p}$ is the proton helicity, and it is expressed as

$$
\frac{d \Delta \sigma^{\lambda_{\ell}}}{d x d y}=\frac{G_{F}^{2}}{\pi\left(1+Q^{2} / M_{W}^{2}\right)^{2}} \frac{Q^{2}}{x y}\left[-\lambda_{\ell} x y(2-y) g_{1}-(1-y) g_{4}-x y^{2} g_{5}\right],
$$

for the CC process by neglecting $M^{2} / Q^{2}$ correction terms. Here, $\lambda_{\ell}$ is the lepton helicity. In the parton model, the leading-twist structure functions $g_{1}, g_{4}$, and $g_{5}$ are expressed in terms of the polarized PDFs. The $g_{4}$ and $g_{5}$ are related by the Callan-Gross type relation $g_{4}=2 x g_{5}$ in the LO, and the CC structure functions $g_{1}$ and $g_{5}$ are expressed:

$$
\begin{array}{ll}
g_{1}^{v p}=\Delta \bar{u}+\Delta d+\Delta s+\Delta \bar{c}, & g_{1}^{\bar{v} p}=\Delta u+\Delta \bar{d}+\Delta \bar{s}+\Delta c, \\
g_{5}^{v p}=\Delta \bar{u}-\Delta d-\Delta s+\Delta \bar{c}, & g_{5}^{\bar{v} p}=-\Delta u+\Delta \bar{d}+\Delta \bar{s}-\Delta c .
\end{array}
$$

It is important that the $g_{1}$ structure functions directly probe the flavor singlet distribution: $\Delta \Sigma(x)=g_{1}^{(v+\bar{v}) p}=\Delta u+\Delta \bar{u}+\Delta d+\Delta \bar{d}+\Delta s+\Delta \bar{s}+\Delta c+\Delta \bar{c}$. Therefore, the quark spin content issue could be clarified by the neutrino scattering although the measured $x$ range is limited. In addition, combining the $g_{5}$ functions for the proton, we obtain $g_{5}^{v p}+g_{5}^{\bar{v} p}=-\left(\Delta u_{v}+\Delta d_{v}\right)-(\Delta s-\Delta \bar{s})-(\Delta c-\Delta \bar{c})$. The $g_{5}$ functions are important for determining the polarized valence-quark distributions.
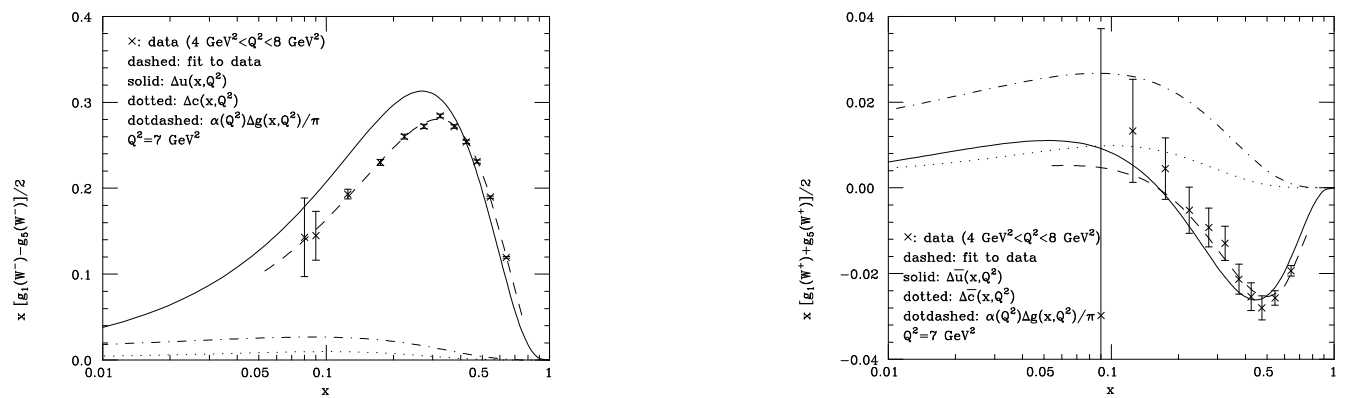

FIGURE 3. Feasibility studies for a neutrino factory [14].

Expected $x\left(g_{1}^{\bar{v} p}-g_{5}^{\bar{v} p}\right) / 2$ and $x\left(g_{1}^{v p}+g_{5}^{v p}\right) / 2$ are shown.

Feasibility is studied for the European neutrino factory in Ref. [14], and some results are shown in Fig. 3. Eight-year running of the neutrino factory with the butanol target is 
assumed for estimating the errors. As shown in the figure, $\Delta u$ and $\Delta \bar{u}$ are the dominant contributions to the combinations $g_{1}^{\bar{v} p}-g_{5}^{\bar{v} p}$ and $g_{1}^{v p}+g_{5}^{v p}$, respectively, and they should be determined by the polarized reactions. However, luminosity has be increased as much as possible for accurate measurements.

A recent HERMES analysis indicates a slightly positive $\Delta s(x)$ at small $x$ [4] in contrast to the parametrization results in Fig. 2. On the other hand, the polarized strangeness $\Delta s$ could be investigated by other neutrino reactions [4]. In elastic neutrino scattering, the axial vector form factor $G_{A}\left(Q^{2}\right)$ can be measured. If non-strange contributions are known, the strange part is extracted: $G_{A}^{s}\left(Q^{2} \rightarrow 0\right)=\Delta s$. At this stage, the analysis of BNL734 data indicates that $G_{A}^{s}$ is consistent with zero. However, there is a proposal to measure it at Fermilab by the FINeSE project [4]. We expect that the strange spin will be clarified by $G_{A}$ as well as the DIS experiments.

\section{NEUTRINO-NUCLEUS SCATTERING}

Nuclear modification of the PDFs is investigated in lepton DIS and high-energy hadron reactions. There are two major parametrizations, EKRS [15] and HKM [16], for nuclear PDFs. Current situation of the HKM studies is shown for the ${ }^{40} \mathrm{Ca}$ nucleus in Fig. 4, where $w(C a, x)$ indicates nuclear modification. A $\chi^{2}$ analysis has been made by using the data on the structure-function ratios $F_{2}^{A} / F_{2}^{A^{\prime}}$ and Drell-Yan cross-section ratios $\sigma_{D Y}^{p A} / \sigma_{D Y}^{p A^{\prime}}$. The nuclear PDFs are expressed by a

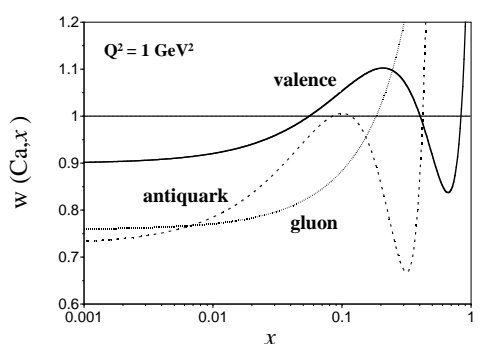

FIGURE 4. Nuclear modification of the PDFs [16]. number of parameters, which are then determined by the $\chi^{2}$ analysis with the data. The valence-quark distributions are determined well in the medium- $x$ region. Because the nuclear modification is negative in this region as shown in Fig. 4 , it is cancelled by the positive one at $x \approx 0.2$ so as to satisfy the charge and baryon-number conservations. However, these conservations do not pose a strong constraint at small $x$, so that the small- $x$ modification is not obvious for the valencequark distributions, and it should be tested by future neutrino DIS measurements. The antiquark distributions are fixed by the observed $F_{2}$ shadowing at small $x$ and the DrellYan data at $x \sim 0.1$; however, the medium- $x$ behavior is not obvious unless new data are obtained. It is difficult to determine the nuclear gluon distributions at this stage.

Because the iron target has been used in neutrino scattering, there are already neutrino-nucleus scattering data. However, it is not possible to investigate nuclear modification due to the lack of accurate deuteron data. At future neutrino facilities, proton and deuteron measurements will become possible, so that the nuclear modification could be investigated. In particular, the $F_{3}$ structure function is specific in the neutrino reaction. Although the $F_{2}$ shadowing is investigated well in the electron and muon scattering, $F_{3}$ shadowing has not been studied at all. The function $F_{3}$ provides information on the valence-quark distribution: $\left(F_{3}^{v N}+F_{3}^{\bar{v} N}\right)_{C C} / 2 \cong u_{v}+d_{v}$. The NuMI project [17] and ultimately the neutrino factories will provide data for the difference 
between the $F_{2}$ and $F_{3}$ shadowing modifications, so that this issue will become clear in future.

We comment on a possible nuclear modification of the longitudinal-transverse structure function ratio $R$. It is sometimes called "HERMES effect". The effect was suggested by the HERMES collaboration in 2000 [18]; however, it was not observed in a CCFR/NuTeV analysis of neutrino data [19] and also in a subsequent HERMES reanalysis with careful radiative corrections [18]. Nonetheless, there could be a nuclear modification at large $x$ with small $Q^{2}$, which is not the observed region by these experiments. A physics origin is the admixture of longitudinal and transverse nucleon structure functions in a nucleus due to nucleon Fermi motion [20]. Such an effect could be investigated by JLab experiments [21] and possibly by future neutrino reactions.

\section{$\sin ^{2} \theta_{\mathrm{W}}$ anomaly from a nuclear physicist's point of view}

The NuTeV collaboration reported anomalously large weak mixing angle: $\sin ^{2} \theta_{W}=$ $0.2277 \pm 0.0013$ (stat) \pm 0.0009 (syst) $[6,22]$ in comparison with a global analysis result $\sin ^{2} \theta_{W}=0.2227 \pm 0.0004$ without neutrino-nucleus scattering data [23]. In the WG2 of this workshop, there are discussions on the $\mathrm{NuTeV}$ result and future experimental studies by parity-violating DIS and Møller scattering [6]. Although there may be new physics [24] behind this difference, it is more natural to seek a mechanism in nuclear corrections of the target iron.

The Paschos-Wolfenstein relation, $R^{-}=\left(\sigma_{N C}^{v N}-\sigma_{N C}^{\bar{v} N}\right) /\left(\sigma_{C C}^{v N}-\sigma_{C C}^{\bar{v} N}\right)=\frac{1}{2}-\sin ^{2} \theta_{W}$, plays an important role for extracting $\sin ^{2} \theta_{W}$ from neutrino and antineutrino scattering data. This relation is valid for the isoscalar nucleon, but there are four correction factors in a nucleus: $R_{A}^{-}=\frac{1}{2}-\sin ^{2} \theta_{W}+O\left(\varepsilon_{v}\right)+O\left(\varepsilon_{n}\right)+O\left(\varepsilon_{s}\right)+O\left(\varepsilon_{c}\right)$. Here, $O(\varepsilon)$ indicates a correction of the order of $\varepsilon$, and the detailed expressions should be found in Refs. $[25,26,27]$. The correction factors are defined by

$$
\begin{array}{ll}
\varepsilon_{n}(x)=\frac{N-Z}{A} \frac{u_{v}(x)-d_{v}(x)}{u_{v}(x)+d_{v}(x)}, & \varepsilon_{v}(x)=\frac{w_{d_{v}}(x, A)-w_{u_{v}}(x, A)}{w_{d_{v}}(x, A)+w_{u_{v}}(x, A)}, \\
\varepsilon_{s}(x)=\frac{s^{A}(x)-\bar{s}^{A}(x)}{w_{v}(x, A)\left[u_{v}(x)+d_{v}(x)\right]}, & \varepsilon_{c}(x)=\frac{c^{A}(x)-\bar{c}^{A}(x)}{w_{v}(x, A)\left[u_{v}(x)+d_{v}(x)\right]}
\end{array}
$$

where $w_{u_{v}}$ and $w_{d_{v}}$ indicate the nuclear modifications of up- and down-valence quark distributions, and $w_{v}$ is defined by $w_{v}=\left(w_{u_{v}}+w_{d_{v}}\right) / 2$. The function $\varepsilon_{n}$ comes from the non-isoscalar nature of the nucleus, $\varepsilon_{v}$ is related to the nuclear modification difference between $u_{v}$ and $d_{v}$, and $\varepsilon_{s}\left(\varepsilon_{c}\right)$ is proportional to the difference between $s$ and $\bar{s}(c$ and $\bar{c})$. The charm correction is expected to be small. The strange correction is also found to be a small effect according to a NuTeV estimate, and it tends to increase the deviation [27]. The valence-quark correction $\varepsilon_{n}$ was found to be small according to model estimates [25] although it should be tested by future experiments. The isovector correction $\varepsilon_{n}$ was included in the NuTeV analysis. It was later investigated in Ref. [26]; however, NuTeV kinematical effects may reduce such a contribution. Therefore, it seems that the nuclear effects $[25,26,27]$ are not enough for explaining the whole deviation at this stage. 


\section{COMMENTS ON LOW-ENERGY NEUTRINO SCATTERING}

We have discussed high-energy neutrino reactions; however, current long baseline neutrino experiments have been done in the low-energy region. In order to understand the neutrino oscillation parameters in a few percent accuracy, the neutrino cross section should be understood accurately as well [7]. There are two important factors. One is to understand the neutrino interaction with the ${ }^{16} \mathrm{O}$ nucleus, another is to describe the cross sections in both DIS and resonance regions. There is a dedicated workshop for this topic, so that the details are found in its web page [28].

First, nuclear corrections should be accurately taken into account [29]. At high energies, they are expressed in terms of the nuclear PDF modifications. At low energies, the corrections include the effects of nuclear binding, Fermi motion, Pauli exclusion, and nucleon-nucleon $(N N)$ correlation. For example, a final-state nucleon in a neutrino reaction suffers from the exclusion effect due to the existence of other nucleons. Such effects modify the small $Q^{2}$ part of the cross section significantly. If the cross sec- FIGURE 5. Quasi-elastic cross section [29]. tion is shown as a function of neutrino energy, The dashed curve includes exclusion effects. the exclusion effect is typically $8 \%$ as shown in

Fig. 5 [29]. From the figure, it is also obvious that the cross section is not accurately measured, and this fact makes it difficult to determine the oscillation parameters accurately. Furthermore, the $N N$ correlation mechanism gives rise to a large momentum tail beyond the Fermi momentum, and it also modifies the cross section significantly. All of these nuclear corrections should be understood clearly for the precise neutrinooscillation physics.

Second, an appropriate model should be studied for describing the cross section smoothly from the DIS to the resonance region because the neutrino data could contain both contributions. It is shown in Ref. [30] that a simple change of the scaling variable $\left[x_{w}=x\left(Q^{2}+0.624\right) /\left(Q^{2}+1.735 x\right)\right]$ could describe the measured $F_{2}$ structure functions fairly well even in the small $Q^{2}$ region $\left(Q^{2}=0.07,0.22\right.$, and $\left.0.85 \mathrm{GeV}^{2}\right)$. Such a simple prescription could be also applied to the neutrino cross sections for the description in both low- and high-energy regions.

\section{SUMMARY}

Future superbeam and neutrino factories provide us a unique opportunity for investigating nucleon substructure, which cannot be studied by other lepton and hadron probes. Nucleon spin structure will be clarified by the leading-twist structure function $g_{1}$ and $g_{5}$. The valence-quark shadowing will be investigated by the $F_{3}$ structure functions for nuclei. We pointed out that these studies together with low-energy nuclear structure studies affect the long-baseline experiments as nuclear corrections. The hadron-structure studies are important, for example, for finding a quark-gluon plasma signature and any exotic 
signature beyond the current physics framework. The future neutrino facilities should play an important role in establishing the hadron-structure physics.

\section{ACKNOWLEDGMENTS}

S.K. would like to thank Y. Kuno for motivating him to hadron-structure studies at the neutrino factories. He thanks the Elsevier Science for permitting him to quote Figs. 2, 3 , and 5 directly from its publications $[12,14,29]$. He was supported by the Grant-inAid for Scientific Research from the Japanese Ministry of Education, Culture, Sports, Science, and Technology.

\section{REFERENCES}

1. D. Michael, talk at this workshop.

2. C. Albright et al., hep-ex/0008064; M. L. Mangano et al., hep-ph/0105155; Y. Kuno et al., NuFact-J studies, version 1.0 (unpublished).

3. M. Aoki, A. Baldini, and B. Fleming, summary of working group 2 at this workshop. See also http:// www.cap.bnl.gov/nufact03/agenda-wg2.xhtml.

4. W. M. Alberico, R. Tayloe, and Y. Miyachi, talks on strangeness at this workshop.

5. U. K. Yang, F. Sergiampietri, and R. Bernstein, talks on neutrino DIS at this workshop.

6. I. Younus, P. E. Reimer, and J. Yu, talks on $\sin ^{2} \theta_{W}$ at this workshop.

7. G. P. Zeller, C. W. Walter, and K. S. McFarland, talks on low-energy neutrino scattering at this workshop.

8. A. L. Kataev and S. Kumano, J. Phys. G 29, 1925 (2003).

9. R. G. Roberts, The Structure of the Proton, Cambridge University press (1990).

10. Neutrino Physics, edited by K. Winter, Cambridge University press (2000).

11. PDF codes are taken from http://durpdg.dur.ac.uk/hepdata/pdf.html.

12. J. Blümlein and H. Böttcher, Nucl. Phys. B 636, 225 (2002). Other curves in Fig. 2 are taken from M. Glück et. al., Phys. Rev. D 63, 094005 (2001); Y. Goto et. al., Phys. Rev. D 62, 034017 (2000).

13. J. Blümlein and N. Kochelev, Nucl. Phys. B 498, 285 (1997).

14. S. Forte, M. L. Mangano, and G. Ridolfi, Nucl. Phys. B 602, 585 (2001).

15. K. J. Eskola et. al., Nucl. Phys. B 535, 351 (1998); Eur. Phys. J. C 9, 61 (1999).

16. M. Hirai, S. Kumano, and M. Miyama, Phys. Rev. D 64, 034003 (2001); in preparation.

17. J. G. Morfin, J. Phys. G 29, 1935 (2003); S. A. Kulagin, hep-ph/9812532.

18. K. Ackerstaff et al., Phys. Lett. B 475, 386 (2000); A. Airapetian et al., hep-ex/0210067 \& 0210068.

19. U. K. Yang et al., Phys. Rev. Lett. 87, 251802 (2001).

20. M. Ericson and S. Kumano, Phys. Rev. C 67, 022201 (2003). See also G. A. Miller, S. J. Brodsky, and M. Karliner, Phys. Lett. B 481, 245 (2000); G. A. Miller, Phys. Rev. C 64, 022201 (2001).

21. A. Brüll et al., http://www.jlab.org/exp_prog/proposals/99/PR99-118.pdf.

22. G. P. Zeller et. al., Phys. Rev. Lett. 88, 091802 (2002).

23. D. Abbaneo et. al., hep-ex/0112021.

24. S. Davidson et. al., J. High Energy Phys. 02, 037 (2002); W. Loinaz et. al., Phys. Rev. D 67, 073012 (2003) and references therein.

25. S. Kumano, Phys. Rev. D 66, 111301 (2002).

26. S. A. Kulagin, Phys. Rev. D 67, 091301 (2003).

27. K. S. McFarland et. al., Nucl. Phys. B 112, 226 (2002); S. Kovalenko, I. Schmidt, and J.-J. Yang, Phys. Lett. B 546, 68 (2002); W. Melnitchouk and A. W. Thomas, Phys. Rev. C 67, 038201 (2003).

28. http://neutrino.kek.jp/nuint01/; http://nuint.ps.uci.edu/.

29. M. Sakuda, Nucl. Phys. B 112, 109 (2002); E. A. Paschos, J.-Y. Yu, and M. Sakuda, hep-ph/0308130.

30. A. Bodek and U. K. Yang, Nucl. Phys. B 112, 70 (2002). 